\title{
The Neuroprotective Effect of Gugijihwang-Tang on Trimethyltin-Induced Memory Dysfunction in the Rat
}

\author{
Eun-Yee Jung, ${ }^{1}$ Mi-Sook Lee, ${ }^{1}$ Chang Joon Ahn, ${ }^{1}$ Seung-Hun Cho, ${ }^{2}$ \\ Hyunsu Bae, ${ }^{1}$ and Insop Shim ${ }^{1,3}$ \\ ${ }^{1}$ Department of Basic Korean Medical Science, Acupuncture and Meridian Science Research Center, Kyung Hee University, \\ Seoul 130-701, Republic of Korea \\ ${ }^{2}$ Hospital of Korean Medicine, College of Korean Medicine, Kyung Hee University, Seoul 130-701, Republic of Korea \\ ${ }^{3}$ Department of Basic Oriental Medical Science, College of Oriental Medicine, Kyung Hee University, Seoul 130-701, Republic of Korea
}

Correspondence should be addressed to Insop Shim; ishim@khu.ac.kr

Received 21 November 2012; Revised 8 April 2013; Accepted 15 May 2013

Academic Editor: Rong Zeng

Copyright (c) 2013 Eun-Yee Jung et al. This is an open access article distributed under the Creative Commons Attribution License, which permits unrestricted use, distribution, and reproduction in any medium, provided the original work is properly cited.

\begin{abstract}
Gugijihwang-Tang (the herbal formula PM012), a decoction consisting of several herbs including Rehmanniae Radix Preparata, has been widely used as herbal treatment for dementia. In order to investigate the neuroprotective action of this prescription, we examined the effect of Gugijihwang-Tang on learning and memory using the Morris water maze and [F-18]FDG micro PET neuroimaging technique. After injection of trimethyltin (TMT, $8.0 \mathrm{mg} / \mathrm{kg}$, i.p.), which is a potent toxicant that selectively kills cells in the central nervous system, rats were administered Gugijihwang-Tang $(100 \mathrm{mg} / \mathrm{kg}$, p.o.) daily for two weeks, followed by the Morris water maze tasks and [F-18]FDG micro PET neuroimaging. In Gugijihwang-Tang administered TMT-treated rats, they showed improved learning and memory abilities in water maze tasks and glucose metabolism, suggesting that GugijihwangTang plays effectively positive role in the improvement of brain function including learning and memory after TMT-induced neurodegeneration. Taken together, our results suggested that the Gugijihwang-Tang should be useful for developing strategies protecting nervous system and improving brain function.
\end{abstract}

\section{Introduction}

Dementia is a brain disorder that seriously affects a person's ability to carry out daily activities and its causes generally include primary neurodegenerative disorders, with the most prevalent being Alzheimer's disease (AD) [1]. Trimethyltin (TMT) is an organotoxic compound with potent neurotoxicant effects which is regarded as being particularly useful for studying the response to injury on account of the distinct pattern of neuronal degeneration in rodent brain. When rat is treated with TMT, it causes a loss of pyramidal neurons predominantly in the rat hippocampal CA3 region starting 3-4 days later [2-4]. Behaviorally, TMT intoxication is characterized by seizure, self-biting, aggressive behavior, hyperactivity, and impairment of working memory [5-7]. The precise mechanism of selective and delayed neuronal death after TMT administration has not been clarified yet. Intoxication with TMT leads to profound neurodegeneration, behavioral and cognitive deficits in both humans and experimental animals [6]. In rats, TMT induced the loss of pyramidal neurons in the hippocampus at 4 days, which was robust at 14 days after TMT administration [8]. These anatomical and behavioral findings have made TMTintoxicated rats an attractive model for degenerative diseases such as $\mathrm{AD}$, the most common cause of dementia $[9,10]$. Herbal medicines, such as Ginkgo biloba, Ginseng, or Melissa officinalis, have been commonly used as memory or cognition enhancers. The effects of these enhancers have been demonstrated scientifically [11, 12]. Yukmi-jiwang gamibang (YJG) has a significant effect on memory enhancement and the expression of genes associated with not only the prevention of neuronal degeneration but also neuronal growth events [13]. Recent double-blind, placebo-controlled trials have also demonstrated that Yukmi-jiwang gamibang significantly enhances cognitive abilities in normal human subjects [14]. 
TABLE 1: The contents of Gugijihwang-Tang and the amounts of standard materials.

\begin{tabular}{ll}
\hline Herbal Medicines & Ratio $(\%)$ \\
\hline Rehmanniae radix Preparant & $8(28.6 \%)$ \\
Cornii fructus & $4(14.3 \%)$ \\
Lycium chinense & $3(10.7 \%)$ \\
Discoreae radix & $4(14.3 \%)$ \\
Hoelen & $3(10.7 \%)$ \\
Mountain Cortex radix & $3(10.7 \%)$ \\
Alismatis radix & $3(10.7 \%)$ \\
\hline
\end{tabular}

However, there have been few reported studies on accessing learning and memory enhancement triggered by treatment with YJG in dementia animal models. Gugijihwang-Tang (GJT), the herbal formula PM012, is another memory or cognition enhancer which is composed of 7 herbal medicines, including steamed Rehmanniac radix Preparata, Lycium chinense, Dioscoreae radix, Corni fructus, Hoelen, Mountain cortex radix, and Alismatis radix. GJT is composed of 7 herbal medicines which are 6 herbal medicines of Yukmi-jiwang gamibang and Lycium chinense. Thus, we examined the effect of GJT (YJG and Lycium chinense) on learning and memory ability in TMT-induced amnesia rats using the Morris water maze. Furthermore, we examined brain activity using the technique of positron emission tomography (PET) with ${ }^{18} \mathrm{~F}$-2 fluoro-2-deoxy-D-glucose ([F-18]FDG) that allows autoradiographic assessment of radiolabeled 2-deoxyglucose uptake. [F-18]FDG micro PET scan, a functional neuroimaging technique, PET brain imaging has been used for the diagnosis and assessment of cerebrovascular diseases, such as dementia. More recently, [F-18]FDG micro PET scan became available for visualizing brain activity in small rodents $[15,16]$. We hypothesized applying this technique in TMT-induced dementia animal model and memory improving substance (GJT). Thus, we have attempted to measure brain activity changes with the technique in TMT-treated rats.

\section{Materials and Method}

2.1. Animals. Male Sprague-Dawley rats weighting 250$280 \mathrm{~g}$ each were purchased from Samtaco Animal Corp. (Kyungki-do, Republic of Korea). The animals were allowed to acclimatize themselves for at least 7 days prior to the experimentation. The animals were housed in individual cages under light-controlled conditions (12/12 hr light/dark cycle) and at $23^{\circ} \mathrm{C}$ room temperature. Food and water were available ad libitum.

2.2. Preparation of Gugijihwang-Tang (GJT). The contents and the ratio of each component in Gugijihwang-Tang are shown in Table 1.

2.3. Reagents. Trimethyltin chloride (TMT; Sigma-Aldrich Inc., St. Louis, MO, USA) or $0.9 \%$ saline (vehicle) was injected and Gugijihwang-Tang was administered into rats during 2 weeks.

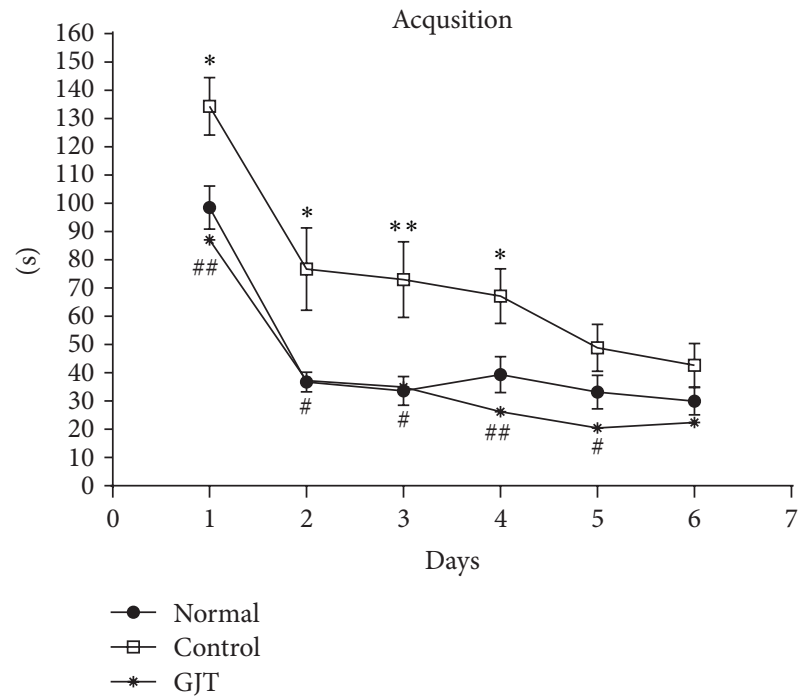

Figure 1: The latency to escape onto the hidden platform during the Morris water maze. The task was performed with 3 trials per day during 6 days for the acquisition test. The values are presented as means \pm S.E.M. ${ }^{*} P<0.05,{ }^{* *} P<0.01$, compared with the normal group; ${ }^{\#} P<0.05,{ }^{\#} P<0.01$, compared with the control group.

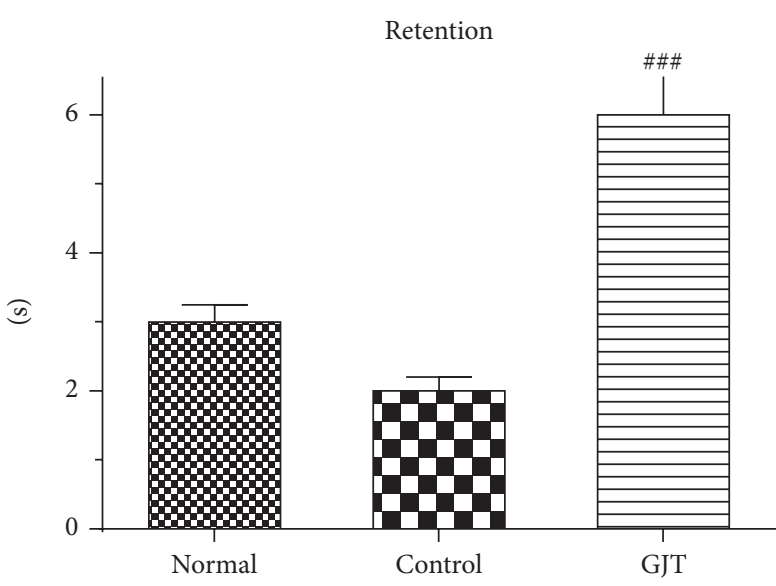

FIGURE 2: Retention performance was tested on the 7th day. The rats received a $1 \mathrm{~min}$ probe trial in which the platform was removed from the pool for retention testing. The values are presented as means \pm S.E.M., ${ }^{\# \#} P<0.001$, compared with the control group.

2.4. Experimental Design. Rats were randomly assigned into three groups ( $n=10$ per group): the normal group (vehicle group), the TMT-lesioned + saline group (the control group), and TMT-lesioned + Gugijihwang-Tang (100 mg/kg treated animals, the GJT group). The rats were injected intraperitoneally (i.p.) with TMT $(8.0 \mathrm{mg} / \mathrm{kg}$, body weight) dissolved in $0.9 \%$ saline and then returned to their home cages. Gugijihwang-Tang (100 mg/kg, p.o.) was dissolved in saline and orally administered for two weeks after TMTinduced neurodegeneration. To verify that TMT causes damage to hippocampal neurons, Nissl staining was performed using a previously described procedure (see Supplementary 
Normal

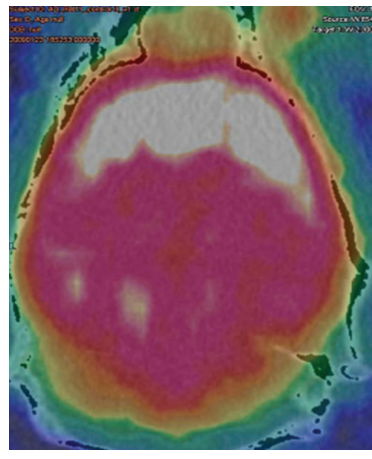

(a)

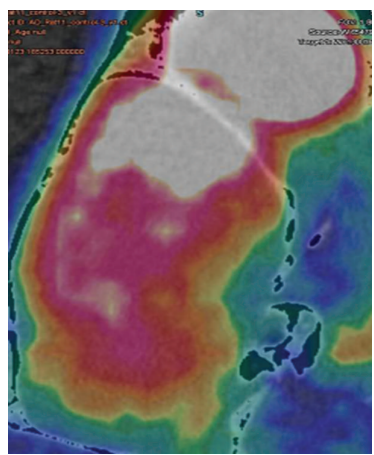

(d)
Control

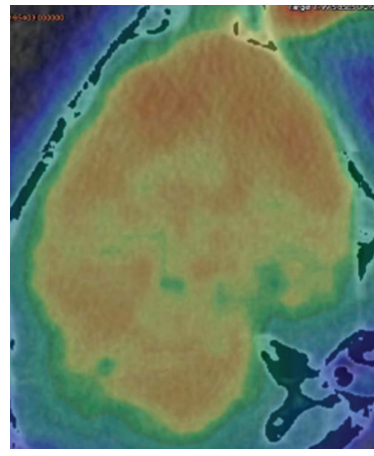

(b)

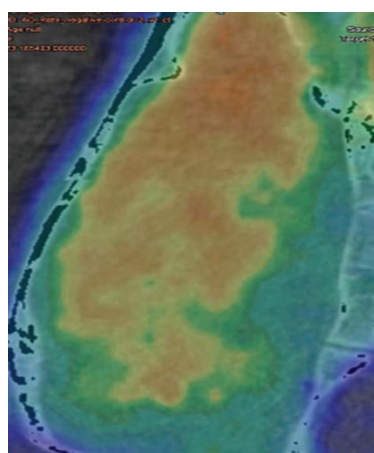

(e)
GJT

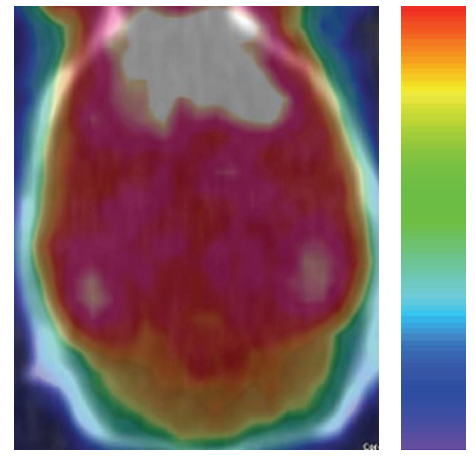

(c)

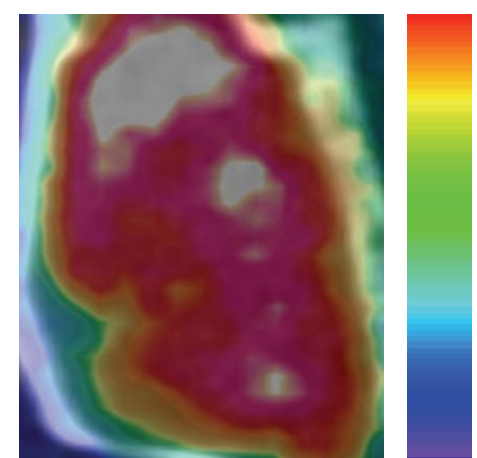

(f)

FIGURE 3: Changes in whole brain glucose metabolism in TMT-induced neurogeneration and treatment of GJT. (a and d) Normal, (b and e) control, and ( $\mathrm{c}$ and $\mathrm{f}$ ) GJT groups, (a to c) axial and (d to f) saggital section of the brain.

Figure 1 in the Supplementary Material available online at http://dx.doi.org/10.1155/2013/542081) [17].

The water maze test was performed for one week and [F18] FDG micro PET neuroimaging from the 15 th day after the injection of TMT.

2.5. Water Maze Test. The swimming pool of the Morris water maze was a circular water tank $200 \mathrm{~cm}$ in diameter and $35 \mathrm{~cm}$ deep. It was filled to a depth of $21 \mathrm{~cm}$ with water at $23 \pm 2^{\circ} \mathrm{C}$. A platform $15 \mathrm{~cm}$ in diameter and $20 \mathrm{~cm}$ in height was placed inside the tank with its top surface being $1.5 \mathrm{~cm}$ below the surface of the water. The pool was surrounded by many cues that were external to the maze. A CCD camera was equipped with a personal computer for the behavioral analysis. Each rat received four daily trials. For 6 consecutive days, the rats were tested with three acquisition tests. They also received retention tests on the 7 th day. For the acquisition test, the rat was allowed to search for the hidden platform for $180 \mathrm{~s}$, and the latency to escape onto the platform was recorded. The animals were trained to find the platform that was in a fixed position during 6 days for the acquisition test, and, then for the retention test, they received a 1 min probe trial in which the platform was removed from the pool. The interval time was $1 \mathrm{~min}$. Performance of the test animals in each water maze trial was assessed by a personal computer for the behavioral analysis (S-mart program, Spain).
2.6. [F-18]FDG Micro PET Scan. All rats were deprived of food for $12-15 \mathrm{~h}$ before the experiments to enhance [F18]FDG uptake in the brain. Each animal was placed on a heating pad in a cage and warmed for at least $30 \mathrm{~min}$ before the [F-18]FDG injection. The temperature of the cages was kept at $30^{\circ} \mathrm{C}$ throughout the uptake period in accordance with an optimized [F-18]FDG uptake protocol [18]. [F-18]FDG (500 $\mu \mathrm{Ci} / 100 \mathrm{~g}$ body weight) was injected through tail vein. After [F-18]FDG injection, radioactivity in rat brain tissue initially rises rapidly. During [F-18]FDG micro PET scans, the animals were kept under isoflurane inhalation anesthesia $(2 \%$ in 100\% oxygen; IsoFlo; Abbott Laboratories).

2.7. Voxel-Based Statistical Analysis. Voxel-based statistical analysis was performed to compare the cerebral glucose metabolism of the group's datasets. The procedure used for SPM analysis of the animal PET data was as previously described in our previous study [18]. Briefly, for efficient spatial normalization, only the brain region was extracted. A study-specific template was then constructed using all the datasets. The PET data was spatially normalized onto a rat brain template and smoothed using a $3 \mathrm{~mm}$ Gaussian kernel. Count normalization was performed. A voxelwise $t$-test between the group's datasets was performed using the Statistical Parametric Mapping 5 program $(P<0.05$, $K>50)$. 
2.8. Behavioral Data Analysis. The data were presented as means \pm S.E.M. Behavioral data (acquisition and retention data) were statistically analyzed by ANOVA testing with repeated measures on the time factor. The origin of the significant effects was further examined by posthoc comparisons using the LSD technique. The criterion for statistical significance was considered to be $P$ values $<0.05$.

\section{Results}

3.1. Effect of Gugijihwang-Tang on Performance in Water Maze Task. The latency to escape onto the hidden platform during the acquisition trial of the water maze was recorded, and the results are depicted in Figure 1. The escape latency differed among the groups when the results were averaged over all the session. The latency to find the hidden platform of the normal and GJT groups was significantly decreased compared with the control group. TMT severely impaired spatial cognition in the water maze test, and the GJT group ameliorated TMTinduced learning and memory deficits in the water maze.

The total time spent on the platform was used to evaluate the spatial performance of the rat during retention trials. The results of the retention test at the 7 th day are depicted in Figure 2. The time spent around platform among the groups differed. The time spent around the platform was $3.3 \pm 0.84$ in the normal group, $2.3 \pm 0.53$ in the control group, and $6.2 \pm 2.2$ in the GJT group. The normal and GJT groups spent more time around the platform than did the control group. The GJT group showed a significant increase $(P<0.001)$ in retention time compared to the control group (Figure 2).

\section{2. [F-18]FDG Micro PET Neuroimaging in the Brain Changes} in the Whole Brain Glucose Metabolism in TMT-Induced Neurodegeneration and Treatment of GJT. Figure 3 shows changes in glucose metabolism of each group in axial and saggital section of the whole brain. Figure 4 summarizes these changes in graph indicating statistical difference between groups.

Figure 5 shows changes in glucose metabolism of each group in coronal section of the region of the hippocampus. Figure 6 summarizes these changes in graph indicating statistical difference between groups. The control group significantly reduced glucose metabolism, exhibiting a $19 \%$ decrease in [F-18]FDG uptake in the whole brain $(P<0.05)$ and a 30\% decrease in the hippocampus $(P<0.05)$ when compared to the normal group. Treatment with GJT can significantly reverse hypometabolism as shown by increasing [F-18]FDG uptake by $67 \%$ in the whole brain $(P<0.05)$ and $62 \%$ in the hippocampus $(P<0.05)$.

\section{Discussion}

Several studies have demonstrated behavioral abnormalities such as increased seizure susceptibility, aggression, and learning impairment after TMT-induced neurodegeneration, which is consistent with results of this study $[5,19]$. The present study confirms previous findings that TMT treatment induces learning impairment from Morris water maze test. This study thus strongly proposes TMT-treated rat to be

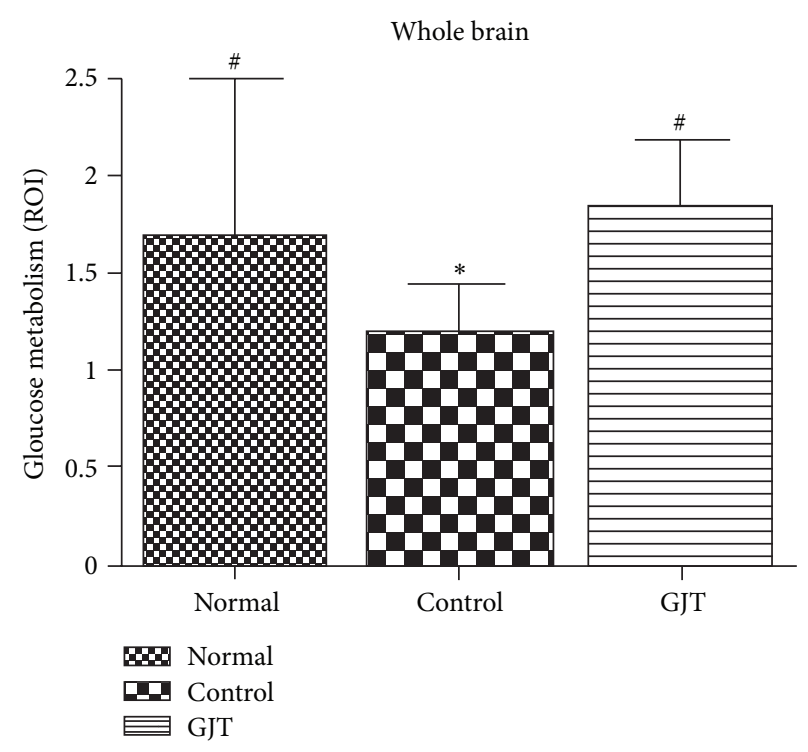

FIGURE 4: Changes in whole brain glucose metabolism in TMTinduced neurogeneration and treatment of GJT. The values are presented as means \pm S.E.M. ${ }^{*} P<0.05$, compared with the normal group, ${ }^{\#} P<0.05$, compared with the control group.

a good model for studying neuronal degeneration. This model may be also useful for elucidating mechanisms underlying resistance to dementia and excite toxic injury in the hippocampus. Other many studies clearly exhibit the functional significance of the hippocampal neurodegeneration by TMT $[2,5,19,20]$. The hippocampus possesses information derived from the associated brain regions that are involved in learning, memory, emotion, and motivation [20]. In TMT exposed rats, GJT treatment improved spatial learning and memory in Morris water maze, suggesting that GJT can reduce spatial deficits on the central nervous system after TMT-induced neurodegeneration. The latency of the GJT group to find the hidden platform was significantly decreased on the acquisition testing, suggesting that GJT could improve the acquisition and retention deficits on the Morris water maze testing. Although the precise mechanisms for recovery of such behavioral deficits following the administering of GJT are not clear, this present study collectively demonstrates that GJT ameliorated learning and memory deficits through its neuroprotective effects on the central nervous system, and thus may have beneficial effects on TMT-induced cognitive impairment.

Numerous studies from epidemiological [21, 22] and functional neuroimaging [23] studies indicate that the dysregulation of energy metabolism in the brain is an important causative factor in the developing dementia. PET imaging has been used for the assessment of dementia and can be used in the diagnosis of disease and new therapeutic searching. More recently, [F-18]FDG micro PET scan became available for visualizing brain activity in small rodents $[15,16]$. [F18]FDG micro PET neuroimaging effects from those components in GJT may have been responsible for the protection against TMT-induced neurodegeneration and cognitive deficits shown in the present study. Thus herbal medicine 


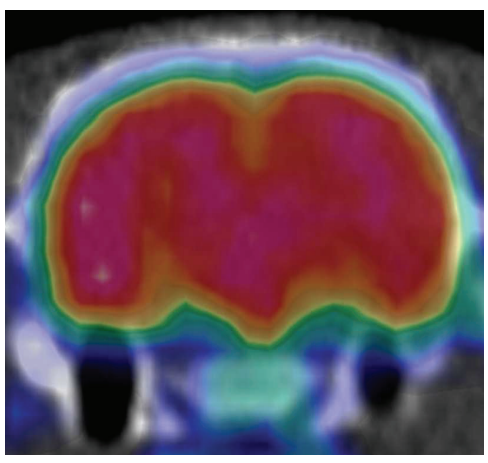

Normal

(a)

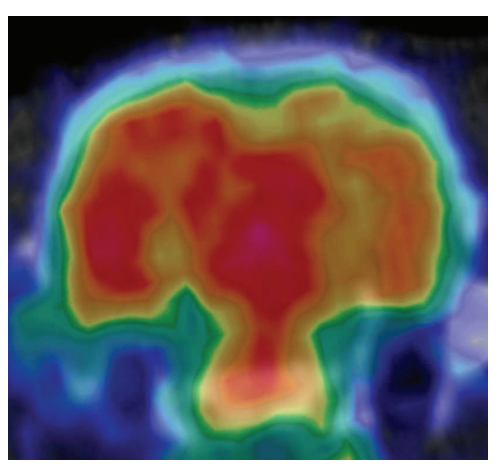

Control

(b)

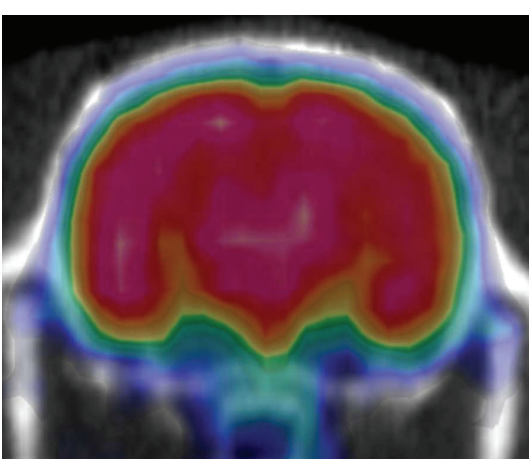

GJT

(c)

FIGURE 5: Changes in the region of the hippocampus glucose metabolism in TMT-induced neurodegeneration and treatment of GJT. (a to c) Coronal section of the brain.

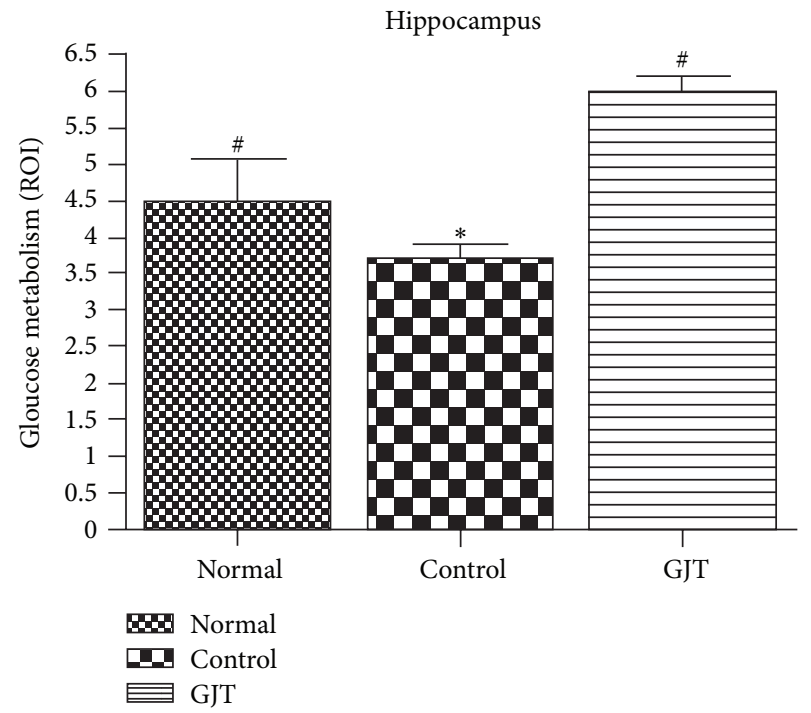

Figure 6: Changes in the region of the hippocampus glucose metabolism in TMT-induced neurodegeneration and treatment of GJT. (a to c) Coronal section of the brain. The value are presented as means \pm S.E.M. ${ }^{*} P<0.05$, compared with the normal group, ${ }^{\#} P<0.05$, compared with the control group.

therapy using GJT in the TMT-induced neurodegeneration brain may have a solid therapeutic potential as a treatment for dementia and amnesia.

\section{Authors' Contribution}

Eun-Yee Jung and Mi-Sook Lee equally contribute to this work.

\section{Acknowledgments}

The authors thank the Korea Basic Science Institute (Chuncheon Center) for the use of facilities. This work was supported by a post-doctoral fellowship Grant from the Kyung
Hee University in 2011 (KHU-20110693) and the Traditional Korean Medicine R\&D Project, Ministry for Health \& Welfare (Grant no. B110025), Republic of Korea.

\section{References}

[1] M. Di Carlo, D. Giacomazza, and P. L. San Biagio, "Alzheimer's disease: biological aspects, therapeutic perspectives and diagnostic tools," Journal of Physics, vol. 24, no. 24, Article ID 244102, 2012.

[2] L. W. Chang and R. S. Dyer, "A time-course study of trimethyltin induced neuropathology in rats," Neurobehavioral Toxicology and Teratology, vol. 5, no. 4, pp. 443-459, 1983.

[3] T. W. Bouldin, N. D. Goines, C. R. Bagnell, and M. R. Krigman, "Pathogenesis of trimethyltin neuronal toxicity," American Journal of Pathology, vol. 104, no. 3, pp. 237-249, 1981.

[4] D. G. Robertson, R. H. Gray, and F. A. De la Iglesia, "Quantitative assessment of trimethyltin induced pathology of the hippocampus," Toxicologic Pathology, vol. 15, no. 1, pp. 7-17, 1987.

[5] N. Ishida, M. Akaike, S. Tsutsumi et al., "Trimethyltin syndrome as a hippocampal degeneration model: temporal changes and neurochemical features of seizure susceptibility and learning impairment," Neuroscience, vol. 81, no. 4, pp. 1183-1191, 1997.

[6] R. S. Dyer, T. J. Walsh, W. F. Wonderlin, and M. Bercegeay, "The trimethyltin syndrome in rats," Neurobehavioral Toxicology and Teratology, vol. 4, no. 2, pp. 127-133, 1982.

[7] P. H. Ruppert, T. J. Walsh, L. W. Reiter, and R. S. Dyer, “Trimethyltin-induced hyperactivity: time course and pattern," Neurobehavioral Toxicology and Teratology, vol. 4, no. 2, pp. 135139, 1982.

[8] Y. Liu, H. Imai, M. Sadamatsu, K. Tsunashima, and N. Kato, "Cytokines participate in neuronal death induced by trimethyltin in the rat hippocampus via type II glucocorticoid receptors," Neuroscience Research, vol. 51, no. 3, pp. 319-327, 2005.

[9] V. Corvino, E. Marchese, F. Michetti, and M. C. Geloso, "Neuroprotective strategies in hippocampal neurodegeneration induced by the neurotoxicant trimethyltin," Neurochemical Research, vol. 38, no. 2, pp. 240-253, 2013.

[10] M. C. Geloso, V. Corvino, and F. Michetti, "Trimethyltininduced hippocampal degeneration as a tool to investigate neurodegenerative processes," Neurochemistry International, vol. 58, no. 7, pp. 729-738, 2011. 
[11] J. Birks, E. V. Grimley, and M. Van Dongen, "Ginkgo biloba for cognitive impairment and dementia," Cochrane Database of Systematic Reviews, no. 4, Article ID CD003120, 2002.

[12] D. O. Kennedy, A. B. Scholey, L. Drewery, V. R. Marsh, B. Moore, and H. Ashton, "Electroencephalograph effects of single doses of Ginkgo biloba and Panax ginseng in healthy young volunteers," Pharmacology Biochemistry and Behavior, vol. 75, no. 3, pp. 701-709, 2003.

[13] S. Rho, M. Kang, B. Choi et al., "Effects of Yukmijihwang-tang derivatives (YMJd), a memory enhancing herbal extract, on the gene-expression profile in the rat hippocampus," Biological and Pharmaceutical Bulletin, vol. 28, no. 1, pp. 87-93, 2005.

[14] E. Park, M. Kang, J. Oh et al., "Yukmijihwang-tang derivatives enhance cognitive processing in normal young adults: a doubleblinded, placebo-controlled trial," American Journal of Chinese Medicine, vol. 33, no. 1, pp. 107-115, 2005.

[15] M. M. Mirrione, W. K. Schiffer, M. Siddiq, S. L. Dewey, and S. E. Tsirka, "PET imaging of glucose metabolism in a mouse model of temporal lobe epilepsy," Synapse, vol. 59, no. 2, pp. 119-121, 2006.

[16] M. M. Mirrione, W. K. Schiffer, J. S. Fowler, D. L. Alexoff, S. L. Dewey, and S. E. Tsirka, "A novel approach for imaging brainbehavior relationships in mice reveals unexpected metabolic patterns during seizures in the absence of tissue plasminogen activator," NeuroImage, vol. 38, no. 1, pp. 34-42, 2007.

[17] J. Lee, D. Son, P. Lee et al., "Protective effect of methanol extract of Uncaria rhynchophylla against excitotoxicity induced by $\mathrm{N}$ methyl-D-aspartate in rat hippocampus," Journal Pharmacological Sciences, vol. 92, no. 1, pp. 70-73, 2003.

[18] H. S. Swartzwelder, R. S. Dyer, W. Holahan, and R. D. Myers, "Activity changes in rats following acute trimethyltin exposure," NeuroToxicology, vol. 2, no. 3, pp. 589-593, 1981.

[19] B. Earley, M. Burke, and B. E. Leonard, "Behavioural, biochemical and histological effects of trimethyltin (TMT) induced brain damage in the rat," Neurochemistry International, vol. 21, no. 3, pp. 351-366, 1992.

[20] L. P. Lanier and R. L. Isaacson, "Activity changes related to the location of lesions in the hippocampus," Behavioral Biology, vol. 13, no. 1, pp. 59-69, 1975.

[21] J. A. Luchsinger, J. M. Noble, and N. Scarmeas, "Diet and Alzheimer's disease," Current Neurology and Neuroscience Reports, vol. 7, no. 5, pp. 366-372, 2007.

[22] P. Toro, P. Schönknecht, and J. Schröder, “Type II diabetes in mild cognitive impairment and Alzheimer's disease: results from a prospective population-based study in Germany," Journal of Alzheimer's Disease, vol. 16, no. 4, pp. 687-691, 2009.

[23] R. A. Sperling, B. C. Dickerson, M. Pihlajamaki et al., "Functional alterations in memory networks in early alzheimer's disease," NeuroMolecular Medicine, vol. 12, no. 1, pp. 27-43, 2010. 


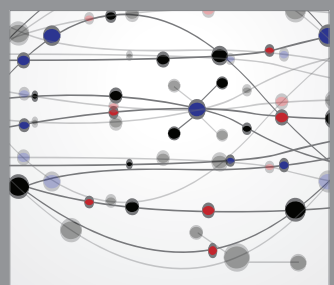

The Scientific World Journal
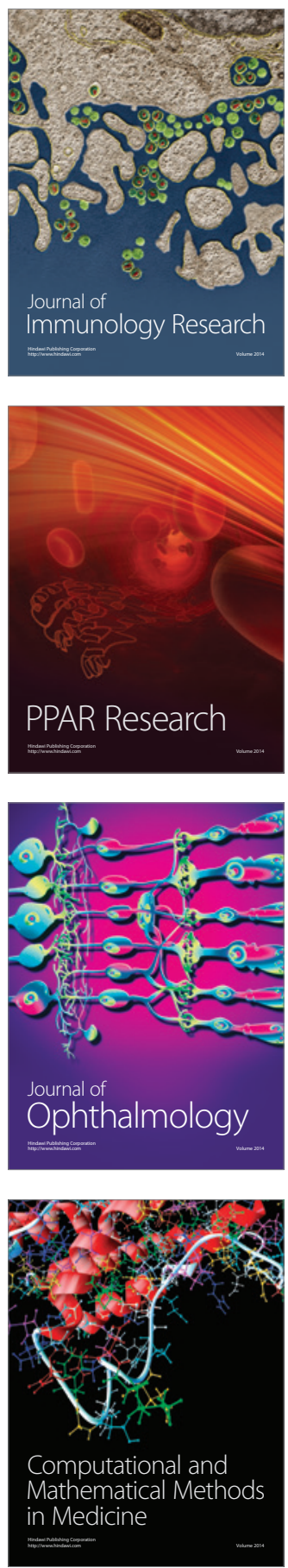

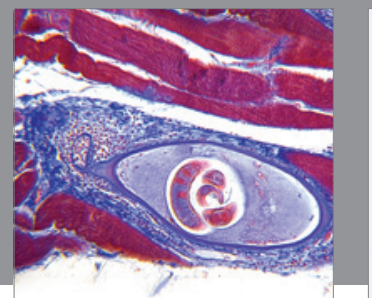

Gastroenterology

Research and Practice
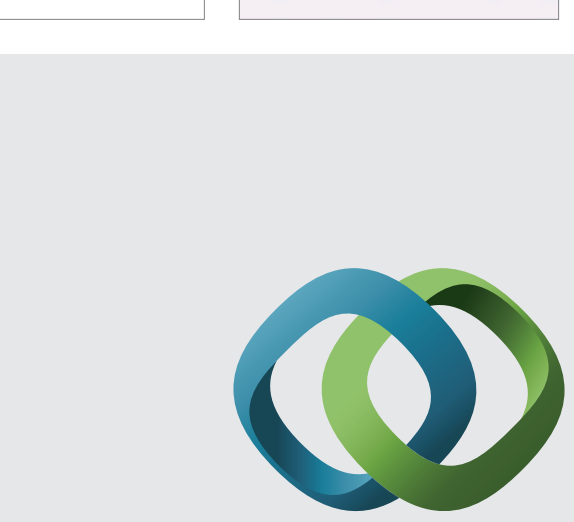

\section{Hindawi}

Submit your manuscripts at

http://www.hindawi.com
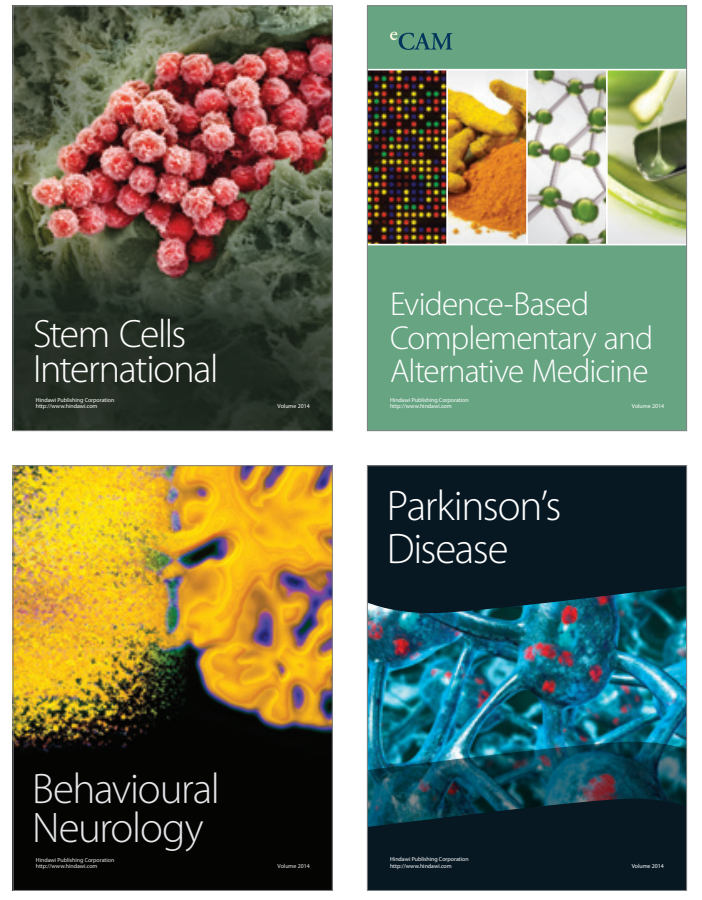
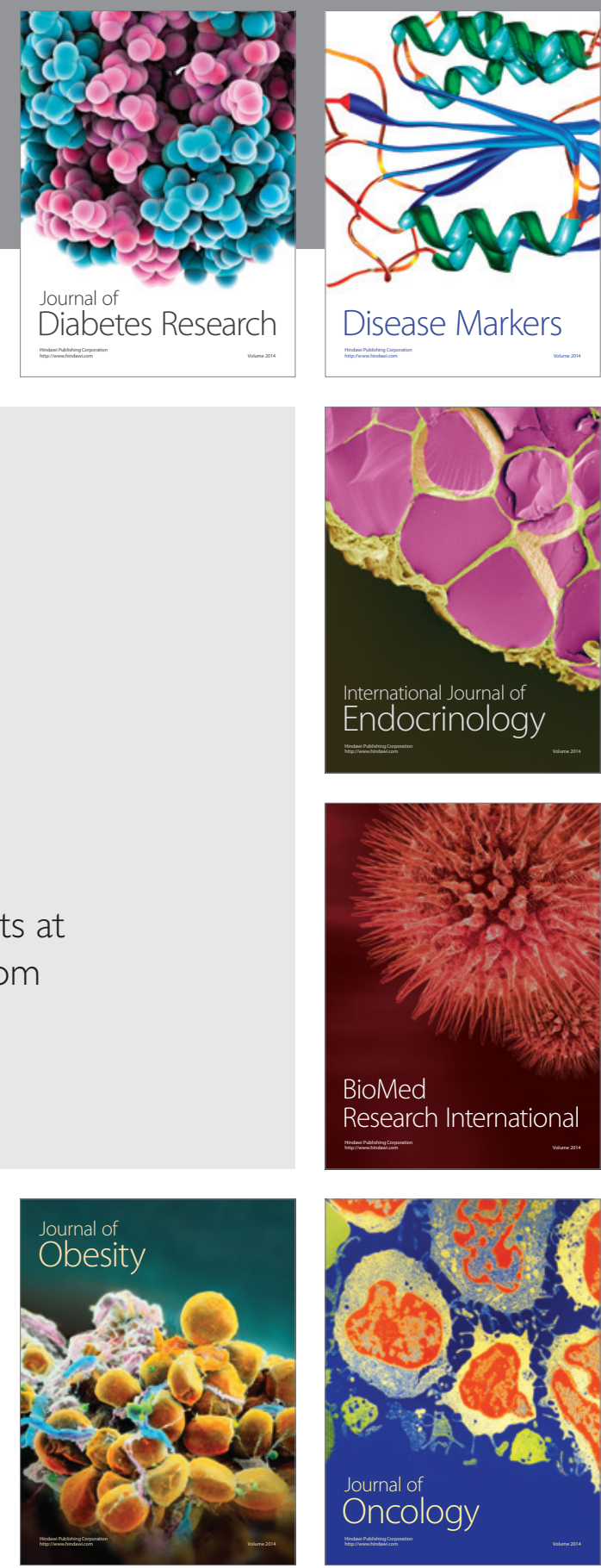

Disease Markers
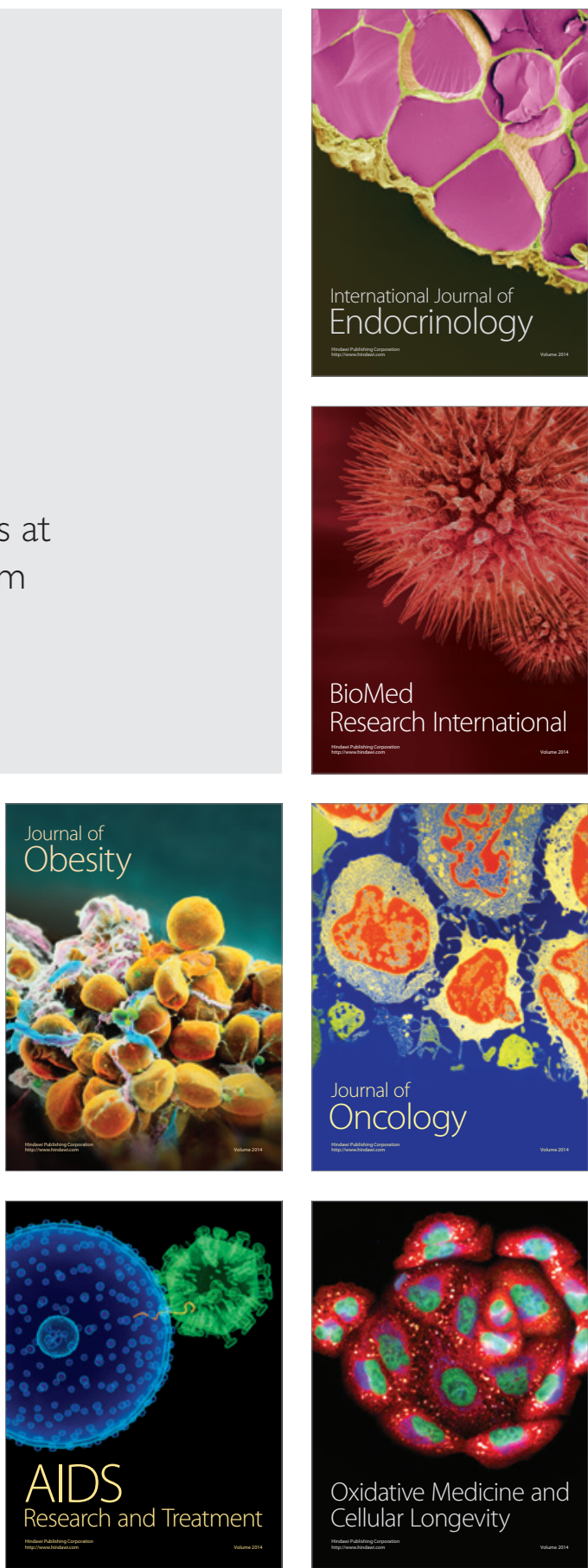